\title{
Clinical Study of 23 Male Patients with Congenital Ventral Penile Angulation without Hypospadias
}

\author{
Ioannis Patoulias, Dimitrios Patoulias*, Konstantinos Farmakis, Maria Kalogirou \\ 1st Department of Pediatric Surgery, Aristotle University of Thessaloniki, GH G. Gennimatas, Thessaloniki, Greece \\ * Corresponding author: M. Alexandrou 3B, Peuka, Thessaloniki, Postal code 57010; e-mail address: dipatoulias@gmail \\ .com
}

Summary: Congenital ventral penile angulation without hypospadias is a rare disease and causes great anxiety to the parents. The aim of our study is the presentation of this disease, especially the indications of surgical treatment and the protocol applied in our clinic. We retrospectively studied 23 male patients aged 2.5 to 7 years old (av 5.2 y) with important penile angulation (over $45^{\circ}$ ) without hypospadias, treated during the past 15 years in our department. In 9 patients the cause was the skin chordee (fibrosis of the ventral part of the prepuce), in 4 the fibrotic fascia (incomplete development of dartos and Buck's fascia) and in 10 the disproportion of the corpora cavernosa. No case of congenital short urethra was reported. In our opinion, the appliance of the algorithm suggested by Donnahoo KK et al. in uncomplicated cases, along with the experience of the surgical team, results in satisfactory treatment and avoidance of complications.

Keywords: Penile angulation without hypospadias; Chordee; Male child; Short urethra

\section{Introduction}

Penile angulation without hypospadias is a stage of penile development at 16 th week of gestation, which gradually disappears from 20 th to 25 th week (1). It can remain in one third of premature neonates and disappears during the first months of life. Congenital penile angulation without hypospadias (CPAwH) less than $45^{\circ}$ is reported in $4-10 \%$ of boys (2). It is usually first observed by the parents.

Incidence of severe CPAwH in Danish children as estimated by Ebbehøj and Metz is $0.37 / 1000$ boys (3). Yachia D applied a vacuum induced erection system, concluding that the incidence rises up to $0.6 \%$ of boys $(4,5)$.

CPAwH with ventral curvature is the most often subtype and concerns about $84 \%$ of all cases, while dorsal $(11 \%)$ and lateral curvature $(5 \%)$ are less frequent (6). Surgical treatment is indicated when ventral curvature exceeds $45^{\circ}(7)$

The aim of our study is to illustrate this rare disease, the surgical indications and the treatment protocol applied in our clinic.

\section{Material and Methods}

During the last 15 years in the 1st Department of Pediatric Surgery, Aristotle University of Thessaloniki we treated 23 boys aged from 2.5 to 7 years old (average $5.2 \mathrm{y}$ ), with congenital penile angulation more than $45^{\circ}$, without hypospadias. We applied the following algorithm, suggested by Donnahoo KK et al (8) (Table 1):
A. Erection test (Figure 1) in order to estimate the degree of the angulation (Gittes and McLaughlin technique) (9).

B. Degloving of the penis after performing a subcoronal circumferential skin incision up to the Buck's fascia and repetition of the erection test. If a straightening of the penis is achieved, we transfer a skin flap from the dorsal to the ventral surface of the penis (button hole or Byars flaps). This transfer is also applied after successful straightening in the following steps.

C. If the angulation persists, excision of the fibrous tissue (malformated dartos and deep fascia of the penis) of the ventral penile surface follows.

D. In cases of still persisting angulation, we conduct a tunica albuginea plication in the position of the greatest curvature. In cases of significant angulation, we place a skin graft on the ventral surface of the corpora cavernosa.

E. In a angulation persists due to a urethral tendering-short urethra, urethral mobilization is needed, combined with construction of neourethra if needed.

F. If the angulation is caused by the disproportion of the corpora cavernosa (corporal rotation and penile disassembly), we longitudinally separate a) the corpus cavernosum from the corpus spongiosum of the urethra starting from the level of the glans and b) the intracavernosal septum. During this procedure, folding of the tunica albuginea of the longer corpus cavernosum respectively to its lateral surface may be needed.

As mentioned above, the operation is completed with the transfer of a skin flap from the dorsal to the ventral surface of the penis. 


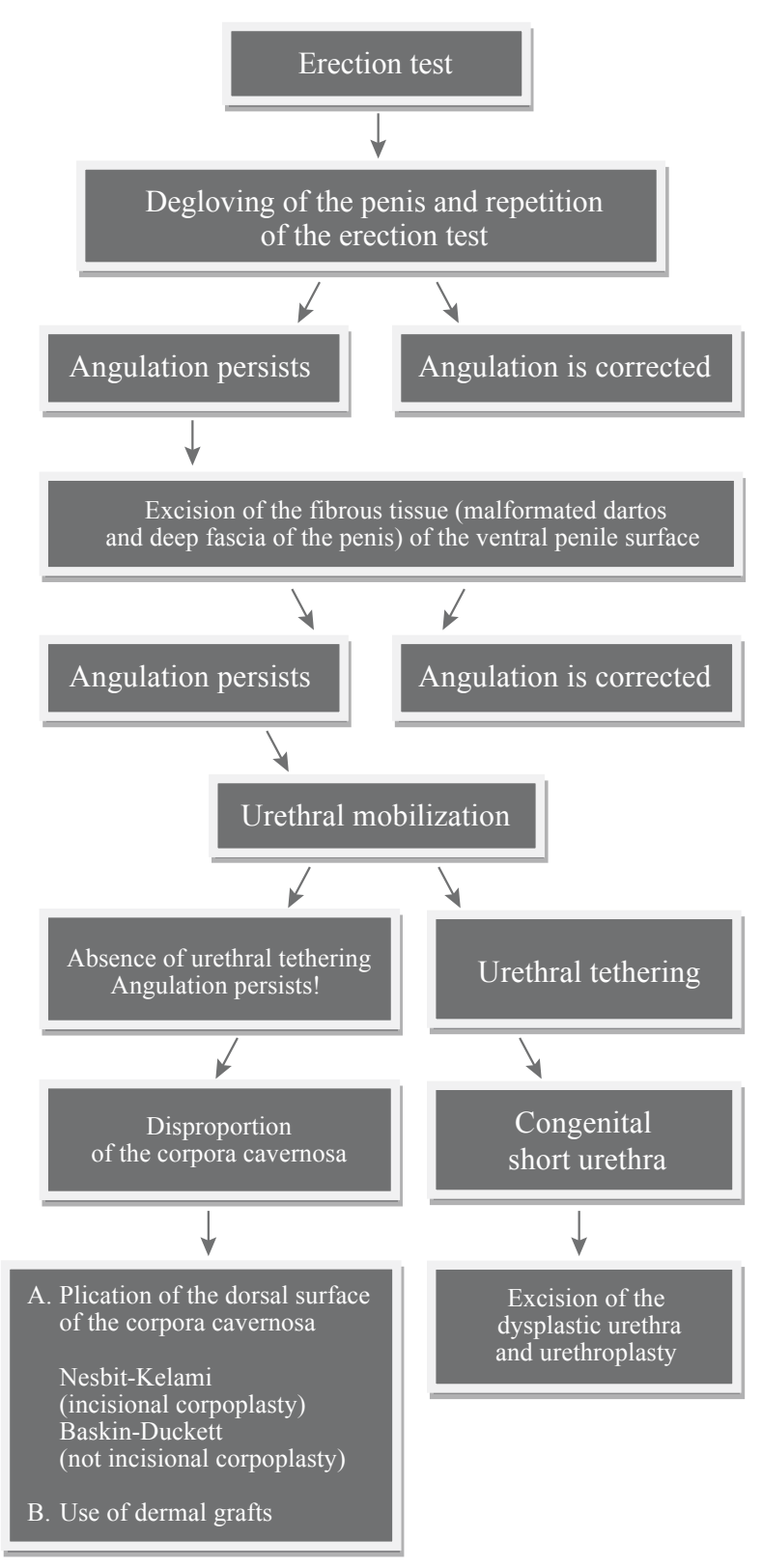

Tab. 1: Algorithm of surgical approach of congenital ventral penile angulation without hypospadias, suggested by Donnahoo KK et al. (8).

\section{Results}

We divided our patients into three groups according to the surgical findings: Group A included 9 patients on which straightening of the penis was achieved by degloving. In Group B, which included 4 patients, excision of the fibrous tissue (malformated dartos and Buck's fascia) from the ventral surface of the penis was conducted. In group C, 10 patients needed a plication of the dorsal surface of the corpora cavernosa. In 6 patients of group $C$ we used

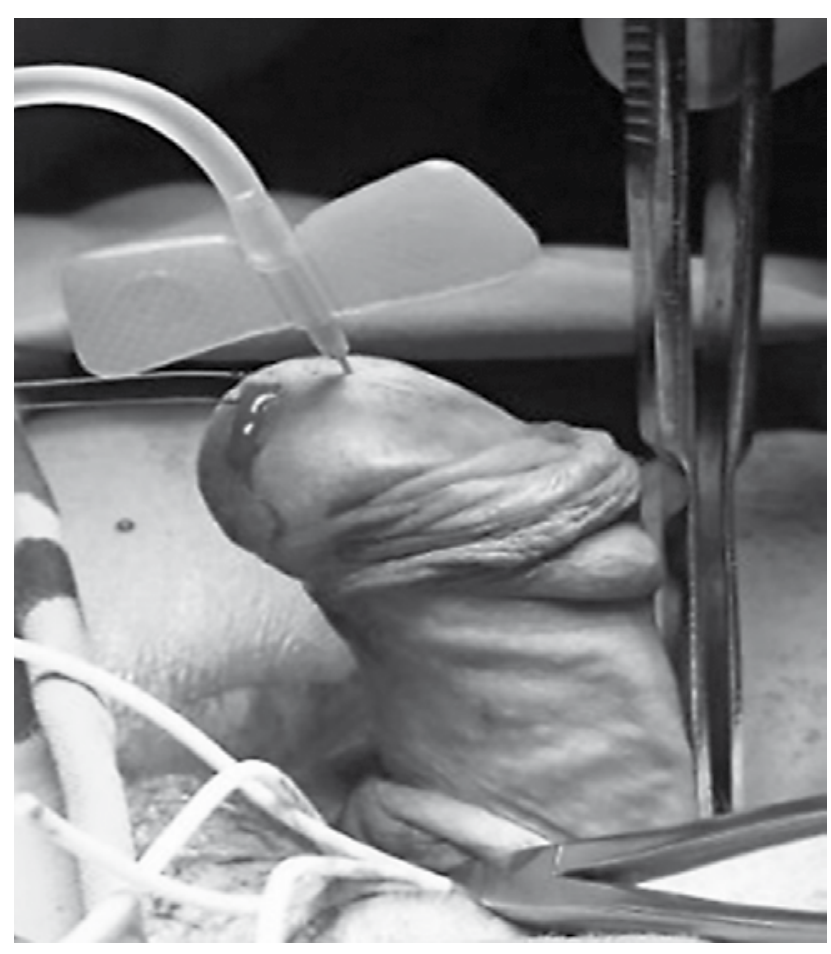

Fig. 1: Erection test. $45^{\circ}$ ventral penile angulation (second patient of group A).

the Nesbit technique and in 4 patients of the same group the Baskin-Duckett technique.

No postoperative complications were reported. The urethral catheter was removed the 2 nd post-op day. Total hospitalization lasted 3-4 days. The moderate edema of the prepuce decreased in the first ten days. In all three groups, the results seven years after the surgical procedure were evaluated as satisfactory (without relapse of the angulation and with penile length within the normal range for the patient's age) and well accepted by parents.

\section{Discussion}

In 1973 Divine and Horton (10) were the first authors that classified CPAwH in three types according to etiology. Type I concerns about a complex malformation of the corpus spongiosum, the Buck's fascia and the dartos. Type II concerns about the Buck's fascia and the dartos and type III only about dartos. A type IV was added 2 years later by Kaplam and Lamm, which is about a disproportion between the dorsal and the ventral surface of the corpora cavernosa (1). A report over type IV was described 7 years later by Kramer, Aydin and Kelalis (2). In 1991 Devine and Blackley et al (11) described a type $\mathrm{V}$ induced by a congenital short urethra or the existence of a fibrous chordee dorsally of the urethra's corpus spongiosum. Type $\mathrm{V}$ is not fully accepted $(7,12,13)$. According to Snodgrass WT (7), type V could be classified as hypospadias variant in the future. 
Donnahoo and Chain et al (8), after studying 87 cases, tried to create an updated classification of CPAwH (Table 2). In our study 9 patients come under type I (38\%), 4 under type $2(18 \%), 10$ under type III (44\%), while there were no patients with type IV of CPAwH.

Tab. 2: Etiology classification of CPAwH by Donnahoo and Chain et al.

\begin{tabular}{|l|l|c|}
\hline Type & Cause & $\mathbf{\%}$ \\
\hline I & $\begin{array}{l}\text { Lack of flexibility of the ventral surface } \\
\text { of the prepuce }\end{array}$ & 32 \\
\hline II & $\begin{array}{l}\text { Fibrosis-malformation of dartos and } \\
\text { Buck's fascia }\end{array}$ & 33 \\
\hline III & $\begin{array}{l}\text { Disproportion between the dorsal and the } \\
\text { ventral surface of the corpora cavernosa }\end{array}$ & 28 \\
\hline IV & Congenital short urethra & 7 \\
\hline
\end{tabular}

The aim of surgical intervention is the creation of a straightened penis during erection, without a proximal transposition of the external urethral orifice, decrease in the penis' length or damage at its structures (corpora cavernosa, urethra, neurovascular bundle complex).

Degloving of the penis was achieved. During the excision of the malformative fibrous tissue (group B) respectively to the ventral penile surface respectively at the level of the dartos, we paid extra attention in order to preserve the corpus spongiosum of the urethra and avoid complications. In group $\mathrm{C}$ we applied incisional corpoplasty (Nesbit's technique) in 6 patients and plicational corpoplasty (Baskin-Duckett's technique) in 4 patients. Using the Nesbit's technique, we incised the fibrous layer in the points of the greatest curvature (along the 11 to 1 o'clock position of the penis), in order to preserve the neurovascular bundle complex $(14,15,16)$.

In order to estimate the accurate number of needed incisions, we followed the principles described by Kelami (14). Before those incisions, we grasped the fibrous layer of the corpora cavernosa using Allis clamps and then conducted the erection test. Provided the desirable result, we incised and sutured the fibrous layer transversally with PDS $3 / 0$ and the knots on the inside of the incision. Plication of the fibrous layer was conducted in 4 patients. Selection of each technique was made by each surgeon. No recurrence caused by sutures transversing the fibrous layer was noticed.

If - in the context of surgical procedure - an extended intervention in the corpora cavernosa is required, this can lead either to a recurrence of the angulation with dorsal orientation or - during the development of the patient - to an iatrogenic shortening of the corpora cavernosa. In this case, it is indicated to make an incision in the ventral surface of corpora cavernosa, respectively to the opposite position of the greatest curvature, and to place skin grafts. Badaway and Morsi evaluated 16 patients who underwent this technique,
10 years later, and found that 14/16 of them had an aligned penis during erection (17).

In cases of short or dysplastic urethra treatment options are:

1. Resection of hypoplastic urethra, excision of underlying fascial tissue and replacement urethroplasty $(18,19,20)$.

2. Division of the mid-portion of hypoplastic urethra and replacement by using a tubularized or island flap or Asopa skin tube $(18,19,20,21)$.

3. Mobilization of hypoplastic urethra and spongioplasty: divergent (Y-shaped) corpus spongiosum along with the hypoplastic urethra, starting from the normal urethra to the tip of glans (22).

If one of the above is applied, but the CPAwH persists, then penile disassembly might be an alternative therapeutic option (23).

Singh S. et al. (21) encountered 26 patients with CPAwH, with successful outcome rising up to $65.6 \%$ of all cases. According to the authors, their results were poorer comparing to those of Donnahoo et al. (8) due to the fact that in their clinical study hypoplastic or dysgenic urethra co-existed in a higher proportion (65.38\% vs. $7 \%$ in Donnahoo's study). They also suggest an interesting classification of the disease based on operative findings. Thus, in our opinion, it plays a significant and catalytic role for the pediatric surgeons in the stepwise management of more complex cases of $\mathrm{CPAwH}$.

We believe that using the algorithm suggested by Donnahoo KK et al. (8), successful treatment without complications can be achieved, especially in the mild to moderate forms of CPAwH. We did not confront serious complications - such as the remaining of the chordee or a fistula -, which are reported in current literature at a rate $7-8 \%$ in the groups $\mathrm{A}-\mathrm{C}$ and $50 \%$ in group D. The absence of patients with type IV CPAwH (dysplastic or short urethra) in our study group led partially to the lower rate of complications.

\section{Conclusions}

1. CPAwH is a rare penile anomaly, cause of great anxiety for the parents. If it is greater than 45 degrees, it must be surgically corrected.

2. Ventral surface of the prepuce, dartos and Buck's fascia are usually involved in its formation.

3. Penile degloving, excision of the malformated fibrous tissue of the ventral surface of the prepuce and straightening of the dorsal surface of the fibrous layer of the corpora cavernosa are sufficient, in order to correct the angulation in $65-92 \%$ of the cases (14).

\section{Conflict of interest}

None of the contributing authors have any conflict of interest, including specific financial interests or relationships and affiliations relevant to the subject matter or materials discussed in the manuscript. 


\section{References}

1. Kaplan GW, Lamm DL. Embryogenesis of chordee. J Urol 1975; 114: 769-72.

2. Kramer S, Aydin G, Kelalis P. Chordee without hypospadias in children. J Urol 1982; 128: 559-61.

3. Ebbehøj J, Metz P. Congenital penile angulation. Br J Urol 1987; 60: 264-6.

4. Yachia D. Early assessment of penile curvatures in infants. J Urol 1991; 145 103-4.

5. Yachia D, Beyar M, Aridogan IA, et al. Incidence of penile curvatures. J Urol 1993; 150: 1478-9.

6. Zachalski W, Krajka K, Matuszewski M. Evaluation of the Treatment of Congenital Penile Curvature Including Psychosexual Assessment. J Sex Med 2015; 12(8): $1828-35$.

7. Snodgrass WT. Management of penile curvature in children. Current Opinion in Urology 2008; 18(4): 431-5.

8. Donnahoo KK, Cain MP, Pope JC, et al. Etiology, management and surgical complications of congenital chordee without hypospadias. J Urol 1998; 160: 1120-2.

9. Gittes RF, McLoughlin AP 3rd. Injection technique to induce penile erection. Urology 1974; 4: 473-4

10. Devine CJ Jr., Horton CE. Chordee without hypospadias. J Urol 1973; 110(2): 264-71.

11. Devine CJ Jr., Blackley SK, Horton CE, Gilbert DA.The surgical treatment of chordee without hypospadias in men. J Urol 1991; 146: 325-8.

12. Baskin LS, Erol A, Li YW, et al. Anatomical studies of hypospadias. J Urol 1998 ; 160: 1108-15.

13. Montag S, Palmer LS, Abnormalities of penile curvature: chordee and penile torsion, ScientificWorldJournal. 2011 Jul 28; 11: 1470-8.
14. Kelami A. Congenital penile deviation and its treatment with Nesbit-Kelami technique. Br J Urol 1987; 60: 261-3.

15. Van Der Horst C, Martínez Portillo FJ, Seif C, et al. Treatment of penile curvature with Essed-Schröder tunical plication: aspects of quality of life from the patients' perspective. BJU Int 2004; 93(1): 105-8.

16. Tang Y-M, Chen S-J, Huang L-G, et al.: Chordee without Hypospadias: Report of 79 Chinese Prepubertal Patients. J Androl 2007; 28(4): 630-3.

17. Badawy H, Morsi H. Long-Term Follow up of Dermal Grafts for Repair of Severe Penile Curvature. J Urol 2008; 180(4): 1842-5.

18. Daskalopoulos EI, Baskin L, Duckett JW, et al. Congenital penile curvature (chordee without hypospadias). Urology 1993; 42(6): 708-12.

19. Bhat A, Sabharwal K, Bhat M, et al., Correction of penile torsion and chordee by mobilization of urethra with spongiosum in chordee without hypospadias, J Pediatr Urol 2014; 10(6): 1238-43.

20. Bhat A, Saxena G, Abrol N. A new algorithm for management of chordee without hypospadias based on mobilization of urethra. J Pediatr Urol 2008; 4(1): 43-50.

21. Singh S, Rawat J, Kureel SN et al. Chordee without hypospadias: Operative classification and its management. Urol Ann 2013; 5(2): 93-8.

22. Jednak R, Hernandez N, Spencer Barthold J, et al. Correcting chordee without hypospadias and with deficient ventral skin: a new technique. BJU Int 2001; 87(6): $528-30$

23. Perovic SV, Djordjevic ML, Djakovic NG, A new approach to the treatment of penile curvature. J Urol 1998; 160(3 Pt 2): 1123-7.

Received: 02/09/2016

Accepted: 20/10/2016 